\title{
Real-time Video-Streaming to Surgical Loupe Mounted Head-Up Display for Navigated Meningioma Resection
}

\author{
Roberto J. DIAZ'1 Jang W. YOON², Robert E. CHEN ${ }^{3,4}$, Alfredo QUINONES-HINOJOSA², Robert E. WHAREN², \\ Ricardo J. KOMOTAR ${ }^{5}$ \\ ${ }^{1}$ Montreal Neurological Institute and Hospital, McGill University, Department of Neurosurgery, Montreal, Canada \\ ${ }^{2}$ Mayo Clinic Florida, Department of Neurological Surgery, Jacksonville, FL, USA \\ ${ }^{3}$ Emory University, School of Medicine, Atlanta, GA, USA \\ ${ }^{4}$ Georgia Institute of Technology, Atlanta, GA, USA \\ ${ }^{5}$ University of Miami, Miller School of Medicine, Department of Neurological Surgery, Miami, FL, USA
}

To watch the surgical videoclip, please visit http://turkishneurosurgery.org.tr/images/20388-video.mp4

\section{ABSTRACT}

Wearable technology interfaces with normal human movement and function, thereby enabling more efficient and adaptable use. We developed a wearable display system for use with intra-operative neuronavigation for brain tumor surgery. The Google glass headup display system was adapted to surgical loupes with a video-streaming integrated hardware and software device for display of the Stealth S7 navigation screen. Phantom trials of surface ventriculostomy were performed. The device was utilized as an alternative display screen during cranial surgery. Image-guided brain tumor resection was accomplished using Google Glass head-up display of Stealth S7 navigation images. Visual display consists of navigation video-streaming over a wireless network. The integrated system developed for video-streaming permits video data display to the operating surgeon without requiring movement of the head away from the operative field. Google Glass head-up display can be used for intra-operative neuronavigation in the setting of intracranial tumor resection.

KEYWORDS: Craniotomy, Google Glass, Image-guided, Neuronavigation, Video-streaming

\section{INTRODUCTION}

$\mathrm{I}$ mage guidance for brain tumor resection has provided neurosurgeons with improved accuracy, thereby optimizing surgical planning, craniotomy design and safety $(2,9)$. Currently, image guidance navigation has to be displayed on a separate screen away from the surgical field on the monitors that are in the operating room. During brain tumor removal, neurosurgeons have to take their eyes away from surgical field and look at the display for the navigation then look back at the field to make sure they are excising tumor infiltrated segments of brain and to ensure the resection margin remains away from tissue with an important functional role. These multiple steps of looking back and forth between the navigation screen and the surgical field can cause delays during tumor resection but also cause error in orientation of the surgeon. This is particularly problematic when other brain areas are overhanging the resection site or when there is bleeding at the resection margin.

In order to enhance the use of navigation images during brain tumor resection, one group has reported projection of 3-D models onto the patient surface during cranial surgery (3). Head-mounted displays have been shown to provide an unobstructed view of the operative field (7).

Corresponding author: Roberto J. DIAZ

E-mail: roberto.diaz@mcgill.ca 
Divergence between the working and viewing direction has been shown to affect efficiency (12). In addition, hand control is influenced by the line of sight when hand position is not in the same axis as the visual guidance system (6). Therefore, we hypothesized that use of Google Glass (Google Inc., Mountain View, CA, USA) display for image-guided tumor resection can enhance the efficiency and accuracy of brain tumor surgery.

In this technical report, we describe the use of a novel image capture device and software on Google Glass to live-stream images to assist resection of a convexity meningioma. Using the device, neuro-navigational images from Stealth Station are captured and transferred wirelessly via password-encrypted network to Google Glass adapted onto wearable surgical loupes.

\section{TECHNIQUE}

\section{Patient}

A 48 year-old female undergoing left parietal craniotomy for a growing meningioma was selected based on requirement for intra-operative image-guided resection. Informed consent was obtained from the patient for use of the surgical loupe mounted video display as an additional display of imageguidance video output.

\section{Image-guidance System Integrated with Medical Image Capture and Streaming Hardware}

The Stealth S7 (Medtronic Inc, Littleton, Massachusetts, USA) image-guidance system was used to register the cranium using scalp fiducial markers after anesthetic induction, positioning, and head-frame fixation. Video from the Stealth S7 VGA output port was converted to HDMI signal. The signal was processed using video compression software protocol executed on a custom-designed hardware chip. After the video signal was detected a video streaming protocol was initiated. The video was transmitted by encrypted wireless communication (WPA-2) onto the Google Glass Head up display. A custom-built software application installed on the Google Glass was used to decode the video signals streamed from the video-streaming component and displays the video on the Google Glass screen. Automated voice commands can be used to launch the software on the Google Glass, thereby maintaining sterility of the surgical field. Image quality was set to stream at $640 \times 360$ pixels, which is at the maximum resolution of the Google Glass. An overview of the system is provided in Figure 1. The interface described was tested using a phantom head (courtesy of Medtronic) prior to use on the patient as described below.

\section{Google Glass Attachment to Surgical Loupes}

The Google Glass hardware was attached to surgical loupes with strong adhesive tape for the surgical procedure (Figure $2 \mathrm{~A}, \mathrm{~B})$. This allowed quick removal in case of surgical need or any discomfort to the surgeon. In order to further optimize the use of the Google Glass with the loupes a detachable adaptor was fabricated for the Google Glass hardware using custom 3-D printing. The Google Glass hardware adds an additional 35 grams of weight to the loupes.

\section{Non-operative Testing}

We performed multiple tests with a phantom head in order to ensure 1:1 image transmission from the Stealth S7 station to the head-up Google Glass display. Accurate navigation was confirmed by using Google Glass display to place the navigation wand on assigned fiducial markers. We also tested the ability of the user to move the navigation wand in order from one surface fiducial marker to another several times without looking at the phantom head. In order to demonstrate rapid image transmission with the capability of allowing the user to either alter self-movement or provide verbal commands to an assistant, we tested the Google Glass display by having the user direct a simulated ventriculostomy placement with only the Google Glass display image as feedback (Video). There was no noticeable delay between the Stealth S7 station and the Google Glass display screen. The operator was able to track the movement of a hand-held wand and view navigation images without turning his head to look at the monitor. Wireless transmission of navigation images to a hand-held device was also tested to demonstrate the image that the surgeon would see on the Google Glass display screen. The accuracy of the navigation is not altered by transmission through the heads-up display system since the image is a duplicate of the output from the Stealth S7 station. The navigation data remain

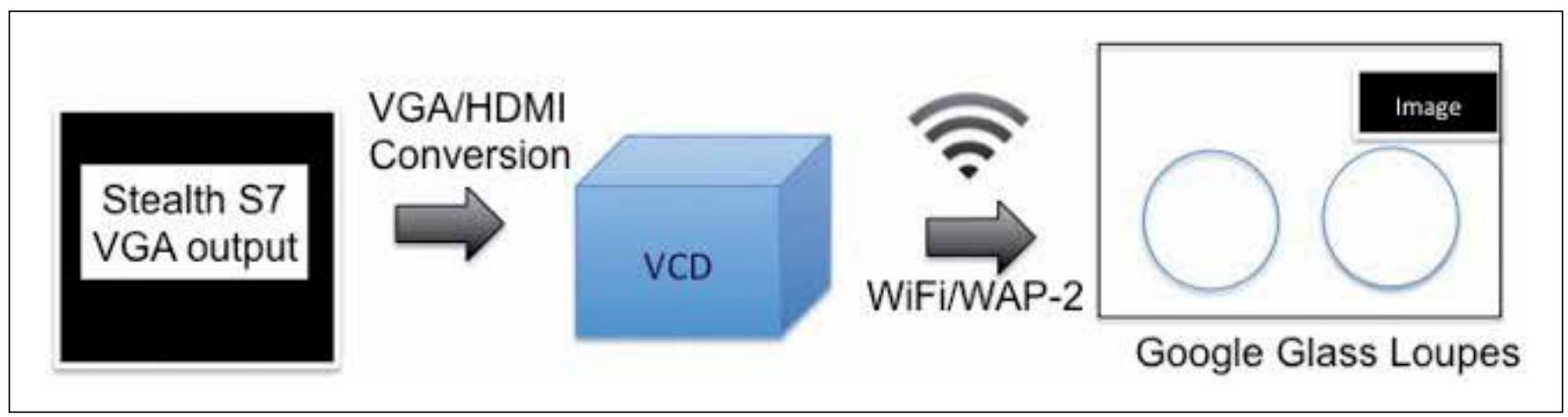

Figure 1: Schematic diagram of the video-capture and streaming system for head-up Google glass display of Stealth S7 imageguidance. VGA-video graphics array, HDMI-high-definition multimedia display, VCD-video capture device, WAP2-Wi-Fi protected access 2. Location of Google Glass image output in relation to loupe magnifying field (circles) demonstrated. 
unaltered when the image capture and streaming system is utilized. Therefore, any navigational inaccuracy is related to the factors that contribute to error in frameless stereotaxy (14).

\section{Intra-operative Testing}

The loupe-mounted head-up display was used to register fiducials with the hand-held navigation wand and 'point merge' feature on Stealth S7. During the surgical procedure we used the hand-held navigation wand and head-up display to plan the incision and to outline the margins of the tumor in order to plan for the bone flap. In addition, the head-up display was used during tumor resection to ensure complete resection of the enhancing dural margin of the meningioma.

\section{Result}

Phantom tasks were completed with the loupe adapted Google Glass device as described above (Figure 3A, B). The surgical procedure for resection of a left parietal convexity meningioma
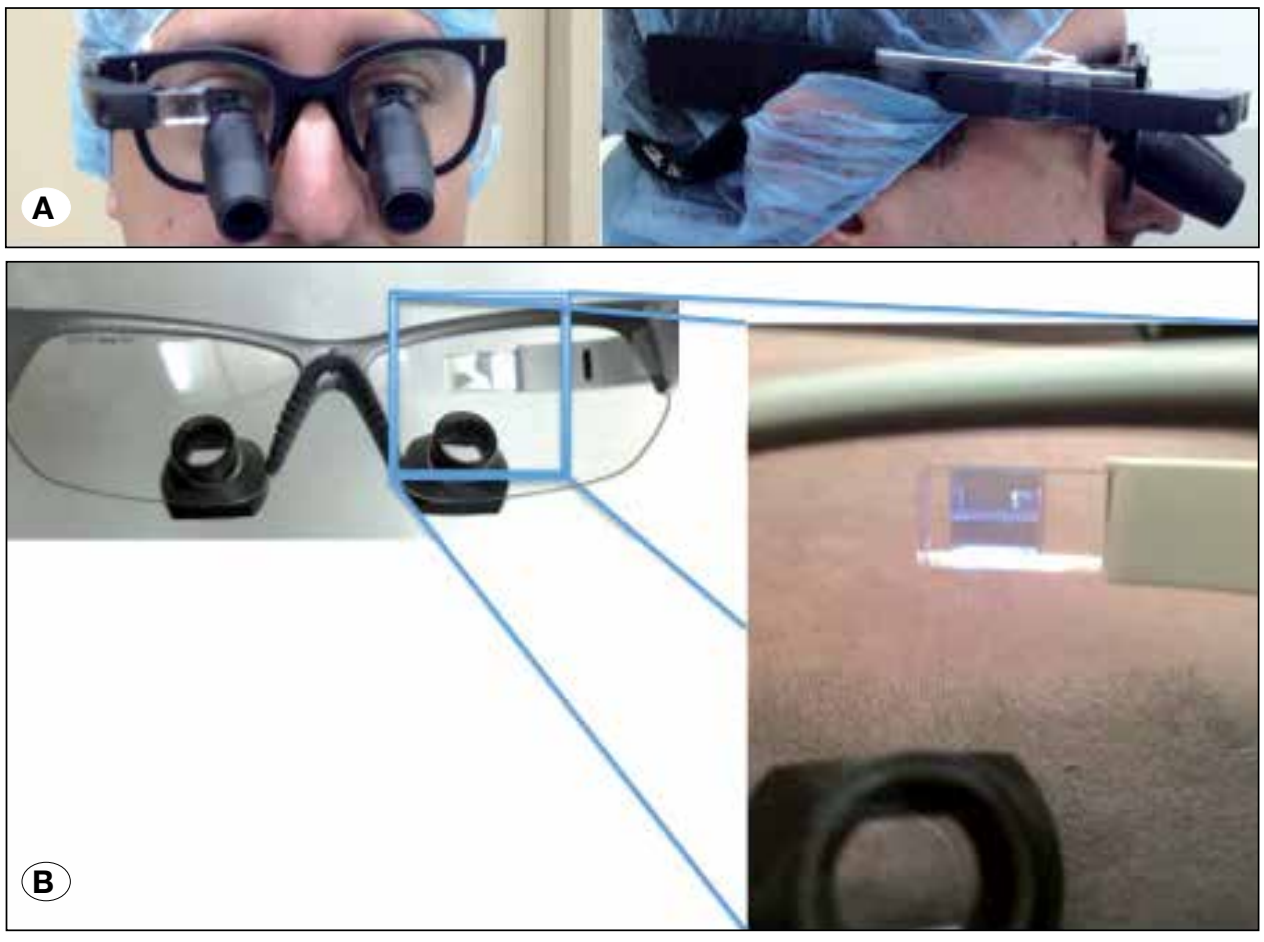

Figure 2: A) Surgeon wearing Google Glass adapted surgical loupes. B) Wide angle and close-up view of surgeon's view point when wearing Google Glass adapted surgical loupes.
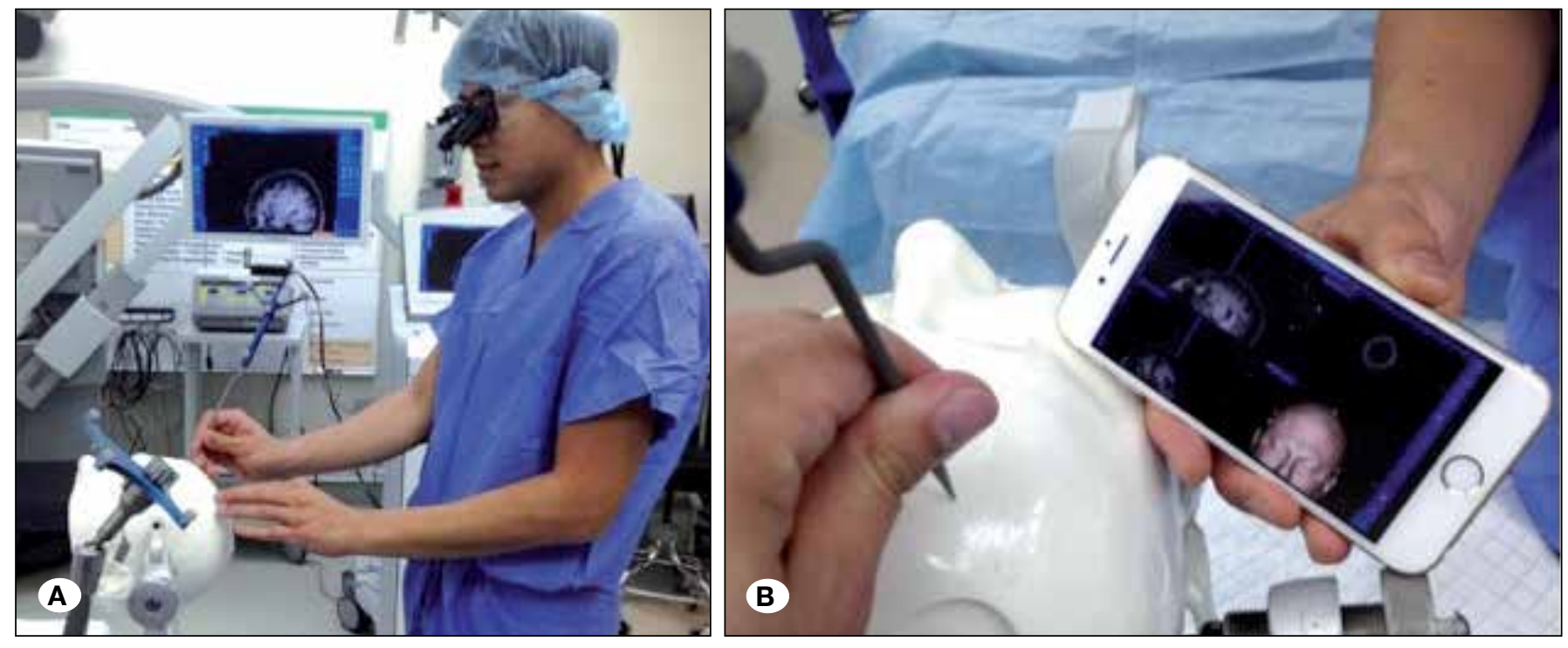

Figure 3: A) Simulated ventriculostomy using a phantom skull with navigation image display on Google Glass adapted surgical loupes. B) Wireless image transmission and display of navigation image on a hand-held device demonstrating the image which would be visible on Google Glass display when using the custom image capture and transmission device. 

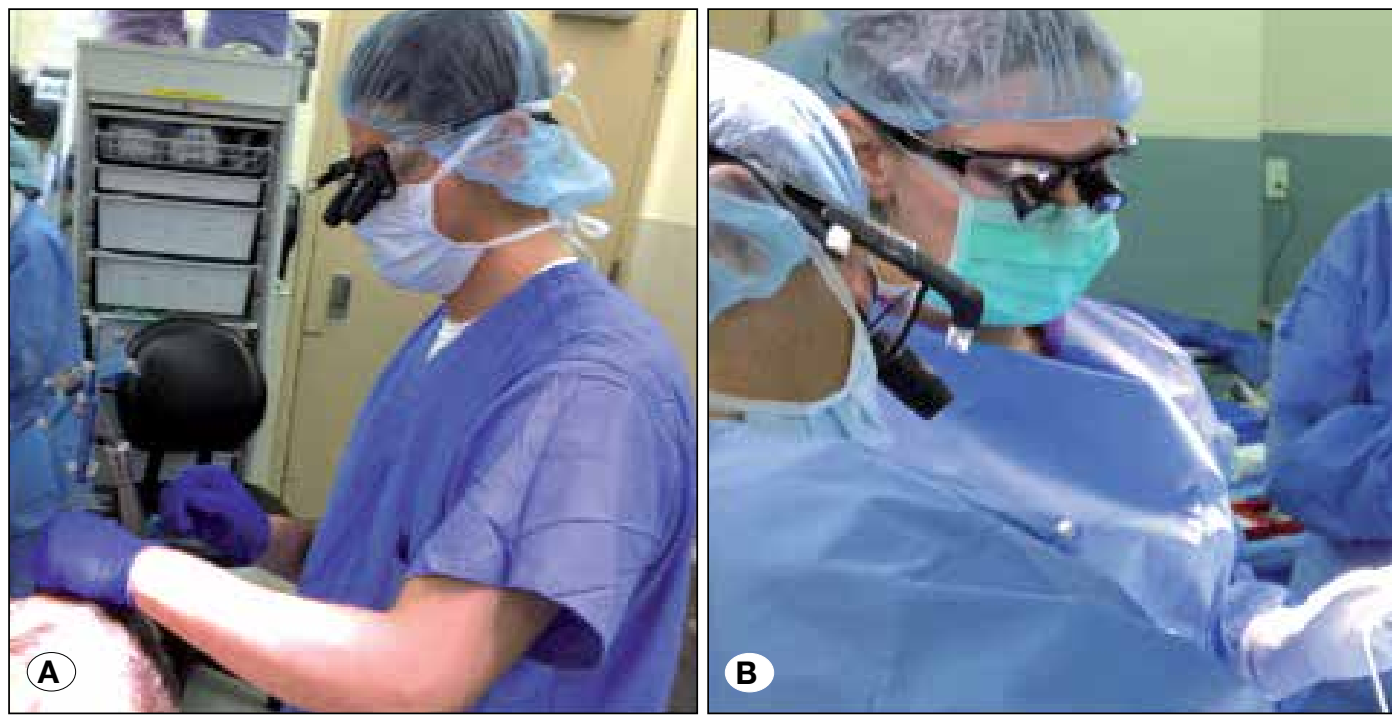

Figure 4: Intra-operative use of Google Glass head-up display during registration $(\mathrm{A})$ and during tumor resection (B).

Table I: Intra-Operative Heads-Up Display Navigation Episodes During Surgery

\begin{tabular}{ccc}
\hline Episode & Duration (sec) & Stage \\
\hline 1. & 312 & Incision and bone flap planning \\
\hline 2. & 18 & Tumor resection \\
\hline 3. & 16 & Tumor resection \\
\hline 4. & 35 & Tumor resection \\
\hline 5. & 9 & Tumor resection \\
\hline 6. & 15 & Tumor resection \\
\hline
\end{tabular}

was completed successfully with no impairment of the surgeon by wearing the loupe-adapted Google Glass device (Figure 4A, B). A detailed description of how the navigation system was used during the surgical case is provided in Table I. Image capture and streaming device (MedCyclops LLC, Jacksonville, FL) was connected to Stealth station and streaming component was automatically launched. Google Glass automatically recognizes a secure WPA-2 wireless network, which is separate from the hospital's wireless network. Upon the surgeon's voice commands, the streaming software is initialized, which allows navigation images to be played in real-time on the Google Glass screen. The Google Glass head-up display added 35-gram of additional weight to the surgical loupes and it did not cause any discomfort for the user. Image quality and transmission speed were adequate for seamless usage during the surgery. In this case we found that the navigation system was used for a longer duration during incision and bone-flap planning than during surgical resection of the dural margin. A Simpson grade 1 resection of the tumor was achieved using the Google Glass display of Stealth S7 navigation images to plan the craniotomy and dural resection (Figure 5).

\section{DISCUSSION}

We report the first wearable head-up display assisted imageguided surgical resection of an intracranial tumor. The Google Glass display, custom software, and custom-built video capture and transmitting device is able to present Stealth S7 navigation images to the line of sight of the operating surgeon in the right upper quadrant of the right eye above the lens component of the surgeon loupes. The image transmission is fast enough for real-time navigation in both a phantom skull surface matching task and simulated ventriculostomy task. Furthermore, we were able to use the device for intra-operative guidance upon resection of the enhancing dural margin of a convexity meningioma. The head-up display allowed intraoperative navigation without the surgeon having to turn his head away from the field during incision of the dural margin. During the surgical procedure a navigation wand was used to interface with the Stealth S7 infrared camera. However, other navigated surgical tools may also be used. The surgeon wearing the device found it comfortable and it did not interfere with performing other tasks during the surgical procedure. In addition, the set-up of the proposed system was simple and intuitive, which permitted plug-and-play capability.

The surgical loupe adapted Google Glass device capable of displaying neuronavigation images provides an important alternative to currently available head-up display navigation technology designed for use with the operative microscope (Microscope Integration, Brain Lab AG). Modern operating microscopes allow navigation images to be superimposed on the microscopic oculars. Use of surgical loupes allows for an appreciation of anatomical relationships, which is lost under high-magnification. The use of the navigation wand and navigated tools is improved by uncoupling the display from the microscope since the microscope and drapes do not interfere with their use. This allows for the use of the Google Glass neuronavigation display in placement of guided ventriculostomy, use during endoscopic navigated procedures, and in minimally invasive stereotactic procedures such as 


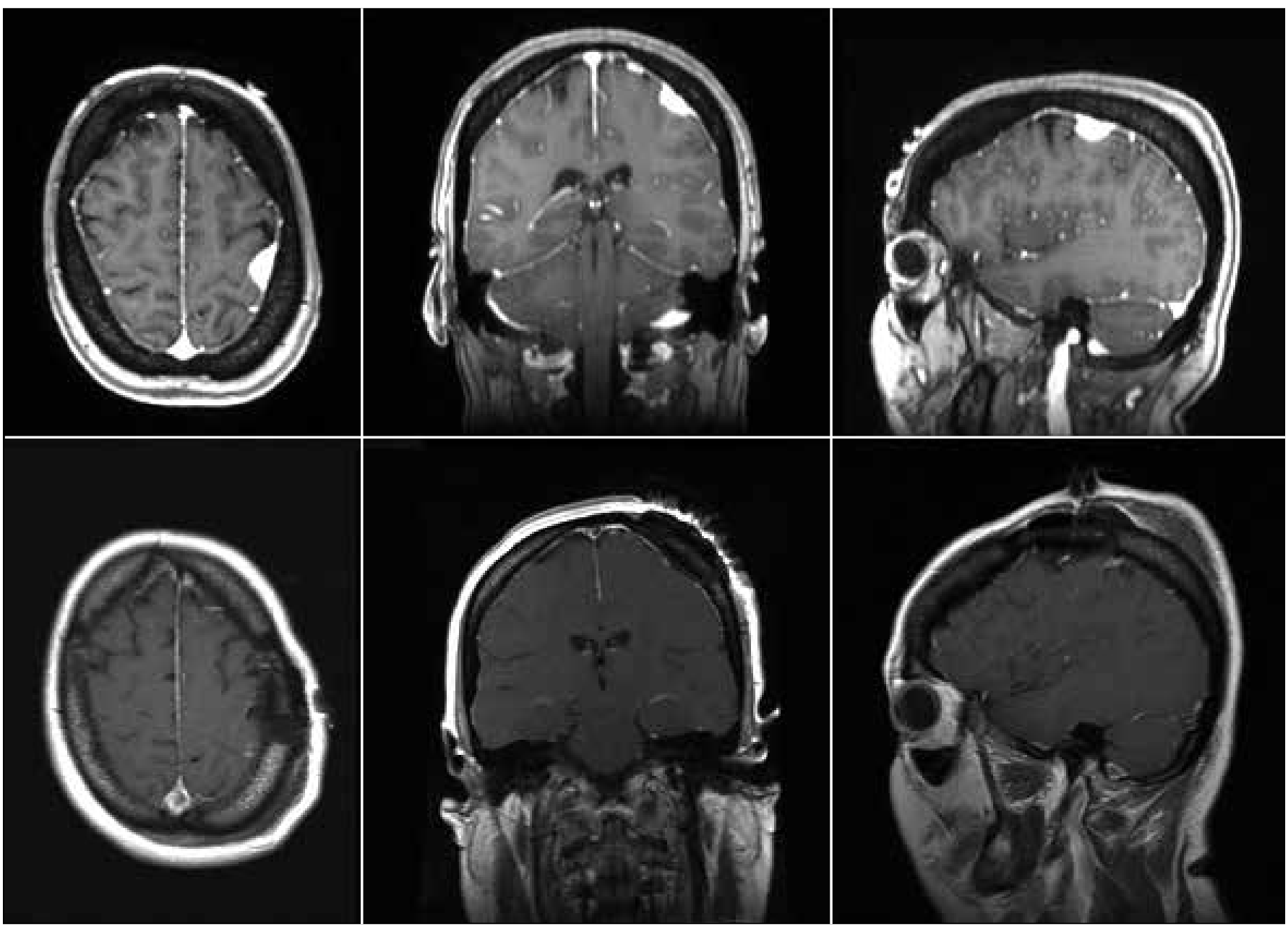

Figure 5: Pre- (top row) and post-operative (bottom row) contrast enhanced T1-weighted magnetic resonance images of left frontal convexity meningioma resected using head-up Google Glass display neuronavigation.

cyst or abscess aspiration. These are all procedures in which the microscope is not used. With the operative microscope display, repeated focusing or autofocusing to acquire the navigation images for a given visual plane is required. With the Google Glass device any focal length on the surgical loupes can be used while preserving the accuracy of the navigation image. Layering of navigation images onto a microscopic view not only creates delays, but it also requires multiple manual steps interrupting the surgeon's concentration and movement (8). One major advantage of using a wearable device instead of the microscope is the improved freedom of movement. A microscope is locked into a position at a certain focal distance and an angle; so that the surgeon has to constantly change his/her view during tumor resection.

It is important to note that the use of LED projectors to display navigation images directly onto the surgical field has been described previously $(3,13)$. Wireless wearable technology offers several advantages over stationary video projectors for use in surgical procedures. Unlike video projection technology where a direct line of sight is required to view projected images on the surgical field, surgeons are able to view navigation images at multiple angles. There is no image distortion or degradation of navigation accuracy since the registration data remains unchanged. In addition, wearable technology allows surgeons to use voice control that allows more natural and seamless integration into the surgical environment.

Google Glass in its current form is not able to provide the necessary magnification and illumination to perform surgery for deep-seated tumors or complex vascular lesions that would typically require the use of the surgical microscope. In the future, wearable technology capable of magnification and virtual object display may permit its use in microsurgical tasks. The Google Glass display does not interfere with the use of a central headlight for improved illumination. However, the additional weight of the display (35 gm) may cause discomfort on the bridge of the nose if worn for long periods of time without rest. If the surgeon experiences discomfort the Google glass display can be removed from the loupes temporarily and then replaced when navigation is required. The system has not been tested within a high-field magnetic resonance imaging (MRI) environment and therefore we do not recommend its use within the magnetic field of an intra- 
operative MRI. The Google Glass display can be cleaned with alcohol or bleach wipes to ensure infection control if splashed with blood accidentally during surgery. No specific sterilization is required for the display since it does not come into contact with the surgical surface.

It is important to note that cognitive attention cannot be simultaneously given to both the operative field under magnification and the head-up navigation screen displayed by Google Glass. Thus, there remains a divergence between the visualized navigation screen and the operative field. It is important to understand this cognitive disconnect so that a task is not conducted under the magnified field to which complete attention is not being given. For example, the user distractibility of a complex Google Glass display that must be processed by the user, such as reading text, has been demonstrated to impair user performance in driving tests (17). Since the Google Glass display is not superimposed on the magnified field the user has to become accustomed to looking up to the right upper quadrant to visualized the navigation image. Learning to adjust focus from the magnified field to the navigation image may take practice and prolonged use may be associated with visual fatigue. We have not used the system continuously for more than 2 hours during which the surgeon has not experienced any adverse effect from the use of the system. In a previous study, a learning curve was observed for adapting to the use of the system for placement of navigated pedicle screws (16).

With wearable technology projected to grow to $\$ 150$ billion by 2026, there will be tremendous technological advancement, which will allow more accurate, interactive and seamless integration of information that surgeons can utilize to enhance surgical experience, efficacy and safety. There are mixed reality wearable devices already demonstrated such as Hololens (Microsoft Corporation, Redmond, Washington, USA) and Magic Leap (Magic Leap, Dania Beach, Florida, USA) with capability to project holographic images onto any surface and allows interaction with projected images using natural commands such as voice and hand motion. Our group is actively testing new wearable devices to adapt these into surgical space to enhance and increase surgical efficacy and safety. Our future plan is to create next generation navigation software and hardware wearable head-up display that can project navigation information in 3-D holographic images directly onto a surgical field and allow surgeons to freely interact with such holographic images in the operating room. Previous work by Quinones-Hinojosa et al. demonstrated that there is an acceptable decrease in the navigation accuracy when the system is moved from frame-based to frameless stereotaxy techniques (11). Our group is planning to create a new algorithm for creating holographic navigation images to account for this expected degradation of navigation accuracy moving onto a head-up display.

Google Glass has been used in multiple medical applications for video-capture and video display (10). Our group was the first to report the utilization of this technology in image-guided spine instrumentation. Utilization of the proposed system resulted in decreased pedicle screw placement time and spine surgeons reported increased focus on the operative task and the patient while the quality of the images were satisfactory for viewing navigation images during surgery (16). The use of head-up fluoroscopy image display has been reported in plastic surgery for visualization of percutaneous fixation of hand fractures (4). Similarly, placement of ultrasound-guided central line with image display on Google Glass has been reported (15).

Here we report the first stereotactic intracranial image guidance broadcast on a head-up display for the purposes of brain tumor resection. The system described is limited by battery life (approximately 2 hours) and the small field of view display of the Google Glass. A battery pack adapter can be adapted to the Google Glass hardware; however, we found that heating of the device can interfere with image display. Future development of image display technology may allow for a larger field of view. We have found that adjusting the magnification on the Stealth S7 display screen can compensate for the small field of view. Integration of verbal command control of the visual display to allow rapid transition between display axes and image type may further facilitate the use of the head-up technology. While Google Glass is no longer commercially available, next generation head-up display and augmented reality technology is in development for the public market and for specialized applications. Furthermore, the video-capture and wireless transmission technology developed for the Google Glass display may be applied in advanced head-up display hardware in the future.

Our work demonstrates the feasibility of the proposed headup display system in cranial neurosurgery. The next phase will be the expansion and inclusion of different types of procedures such as endoscopic surgeries, minimally invasive brain and spine surgeries, surgical oncology and numerous other surgical procedures. It has not escaped our attention that important physiological data such as $\mathrm{pCO} 2$, blood pressure, heart rate, electromyography (EMG) and motor evoked potentials can also be streamed into the visual display of the surgeon to aid in brain tumor surgery. Furthermore, the use of the head-up display to provide the surgeon output from stimulated surface-enhanced Raman scattering (SERS) spectral tissue analysis (5) may facilitate rapid biomarkerguided brain tumor resection.

\section{- CONCLUSION}

A novel video-capture device enabling real-time Google Glass display for image-guided intracranial tumor surgery is presented. The utilization of the proposed system is currently limited to surgical cases in which the microscope is not needed. Further testing of the system in a prospective cohort of tumor patients is anticipated to define the efficiencies provided by the system and to characterize its use with multimodality image display and navigated surgical instruments.

\section{ACKNOWLEDGEMENTS}

We thank Phillip K. Han and Phong Si for technical advice and support. We thank the University of Miami Hospital and 
Medtronic for providing equipment and space for the testing of the device described. The use of Google Glass described in this article has not yet been evaluated and approved by the FDA. The device discussed in this manuscript is only referred to in generic terms. It is the property of MedCyclops.

\section{- DISCLOSURES}

Dr. Yoon is the founder and CEO of MedCyclops. Phillip Han, Robert Chen, and Phong Si are co-founders of MedCyclops. Dr. Wharen is an advisor for MedCyclops.

\section{- REFERENCES}

1. @idtechex: Wearable Technology 2016-2026: IDTechEx. 2016

2. Barnett $\mathrm{GH}$ : The role of image-guided technology in the surgical planning and resection of gliomas. J Neurooncol 42(3): 247-258, 1999

3. Besharati Tabrizi L, Mahvash M: Augmented reality-guided neurosurgery: Accuracy and intraoperative application of an image projection technique. J Neurosurg 123(1): 206-211, 2015

4. Chimenti PC, Mitten DJ: Google glass as an alternative to standard fluoroscopic visualization for percutaneous fixation of hand fractures: A pilot study. Plast Reconstr Surg 136(2): 328-330, 2015

5. Desroches J, Jermyn M, Mok K, Lemieux-Leduc C, Mercier J, St-Arnaud K, Urmey K, Guiot MC, Marple E, Petrecca K, Leblond F: Characterization of a Raman spectroscopy probe system for intraoperative brain tissue classification. Biomed Opt Express 6(7): 2380-2397, 2015

6. Emam TA, Hanna G, Cuschieri A: Ergonomic principles of task alignment, visual display, and direction of execution of laparoscopic bowel suturing. Surg Endosc 16(2): 267-271, 2002

7. Geis WP: Head-mounted video monitor for global visual access in mini-invasive surgery. An initial report. Surg Endosc 10(7): 768-770, 1996
8. Kantelhardt SR, Gutenberg A, Neulen A, Keric N, Renovanz $M$, Giese A: Video-assisted navigation for adjustment of image-guidance accuracy to slight brain shift. Operative Neurosurgery 11(4):504-511, 2015

9. Mahvash M, Boettcher I, Petridis AK, Besharati Tabrizi L: Image guided versus conventional brain tumor and craniotomy localization. J Neurosurg Sci 61(1):8-13, 2017

10. Mitrasinovic S, Camacho E, Trivedi N, Logan J, Campbell C, Zilinyi R, Lieber B, Bruce E, Taylor B, Martineau D, Dumont EL, Appelboom G, Connolly ES Jr: Clinical and surgical applications of smart glasses. Technol Health Care 23(4): 381401, 2015

11. Quinones-Hinojosa A, Ware ML, Sanai N, McDermott MW: Assessment of image guided accuracy in a skull model: Comparison of frameless stereotaxy techniques vs. framebased localization. J Neurooncol 76(1): 65-70, 2006

12. Schier F, Beyerlein S, Gauderer MW: Imaging for endoscopic surgery: New developments applicable to pediatric surgical interventions. Pediatr Surg Int 18(5-6): 459-462, 2002

13. Stadie AT, Kockro RA, Serra L, Fischer G, Schwandt E, Grunert P, Reisch R: Neurosurgical craniotomy localization using a virtual reality planning system versus intraoperative image-guided navigation. Int J Comput Assist Radiol Surg 6(5): 565-572, 2011

14. Wang MN, Song ZJ: Classification and analysis of the errors in neuronavigation. Neurosurgery 68(4): 1131-1143; discussion 1143, 2011

15. Wu TS, Dameff CJ, Tully JL: Ultrasound-guided central venous access using Google Glass. J Emerg Med 47(6): 668675,2014

16. Yoon JW, Chen RE, Han PK, Si P, Freeman WD, Pirris SM: Technical feasibility and safety of an intraoperative head-up display device during spine instrumentation. Int $\mathrm{J}$ Med Robot 13(3), 2017

17. Young KL, Stephens AN, Stephan KL, Stuart GW: In the eye of the beholder: A simulator study of the impact of Google Glass on driving performance. Accid Anal Prev 86: 68-75, 2015 